The University of San Francisco

USF Scholarship: a digital repository @ Gleeson Library | Geschke Center

Nursing and Health Professions Faculty Research

and Publications

School of Nursing and Health Professions

Spring 5-20-2012

\title{
Self-Tracking, Social Media and Personal Health Records for Patient Empowered Self-Care
}

C Paton

Margaret M. Hansen EdD, MSN, RN

University of San Francisco, mhansen@usfca.edu

L Fernandez-Luque

AYS Lau

Follow this and additional works at: http://repository.usfca.edu/nursing_fac

Part of the Medical Education Commons, and the Other Nursing Commons

\section{Recommended Citation}

Paton, C; Hansen, Margaret M. EdD, MSN, RN; Fernandez-Luque, L; and Lau, AYS, "Self-Tracking, Social Media and Personal Health Records for Patient Empowered Self-Care" (2012). Nursing and Health Professions Faculty Research and Publications. Paper 17.

http://repository.usfca.edu/nursing_fac/17 


\title{
Self-Tracking, Social Media and Personal Health Records for Patient Empowered Self-Care
}

\author{
Chris Paton ${ }^{1 *}$, Margaret Hansen ${ }^{2}$, Luis Fernandez-Luque ${ }^{3}$, Annie Y.S. Lau ${ }^{4}$ \\ ${ }^{1 *}$ Corresponding author. National Institute for Health Innovation, University of Auckland, New Zealand. \\ c.paton@auckland.ac.nz \\ ${ }^{2}$ School of Nursing and Health Professions, University of San Francisco, California, USA \\ ${ }^{3}$ Northern Research Institute, Troms $\varnothing$, Norway \\ ${ }^{4}$ Centre for Health Informatics, Australian Institute of Health Innovation, University of New South \\ Wales, Australia
}

\section{Summary}

Objectives: This paper explores the range of self-tracking devices and social media platforms used by the self-tracking community, and examines the implications of widespread adoption of these tools for scientific progress in health informatics.

Methods: A literature review was performed to investigate the use of social media and self-tracking technologies in the health sector. An environmental scan identified a range of products and services which were used to exemplify three levels of self-tracking: self-experimentation, social sharing of data and patient controlled electronic health records. 
Results: There appears to be an increase in the use of self-tracking tools, particularly in the health and fitness sector, but also used in the management of chronic diseases. Evidence of efficacy and effectiveness is limited to date, primarily due to the health and fitness focus of current solutions as opposed to their use in disease management.

Conclusions: Several key technologies are converging to produce a trend of increased personal health surveillance and monitoring, social connectedness and sharing, and integration of regional and national health information systems. These trends are enabling new applications of scientific techniques, from personal experimentation to e-epidemiology, as data gathered by individuals are aggregated and shared across increasingly connected healthcare networks. These trends also raise significant new ethical and scientific issues that will need to be addressed, both by health informatics researchers and the communities of self-trackers themselves.

Keywords: Social Media; Personal Health Records; Public Health Informatics; Self-tracking; Data-mining.

\section{Introduction}

Data from multiple hospital information systems, national disease repositories and thousands of primary care organisations are accumulating at an ever increasing rate. As these data sources are joined together through Health Information Exchanges (HIEs) and national Electronic Health Records (EHR) systems, a vast scientific data-set spanning millions of individuals will finally be available to health informaticians with the promise of new insights into health and disease (1). Exciting as these developments are, there is also a parallel effort, at a significantly more granular level, that will give, not

just scientists, but patients, care-givers and interested members of the public the opportunity to participate in advancing the science of health informatics. 
Consumer health informatics in the past decade has moved from passive patient education to activities that encourage patient participation and engagement in their health care through the use of three key innovations: i) wearable devices, ii) social media websites, and iii) personal health records. These three innovations have enabled the rapid rise of self-tracking communities of patients and health-conscious consumers who generate, store and analyse information about their health status to inform their health-related decision making.

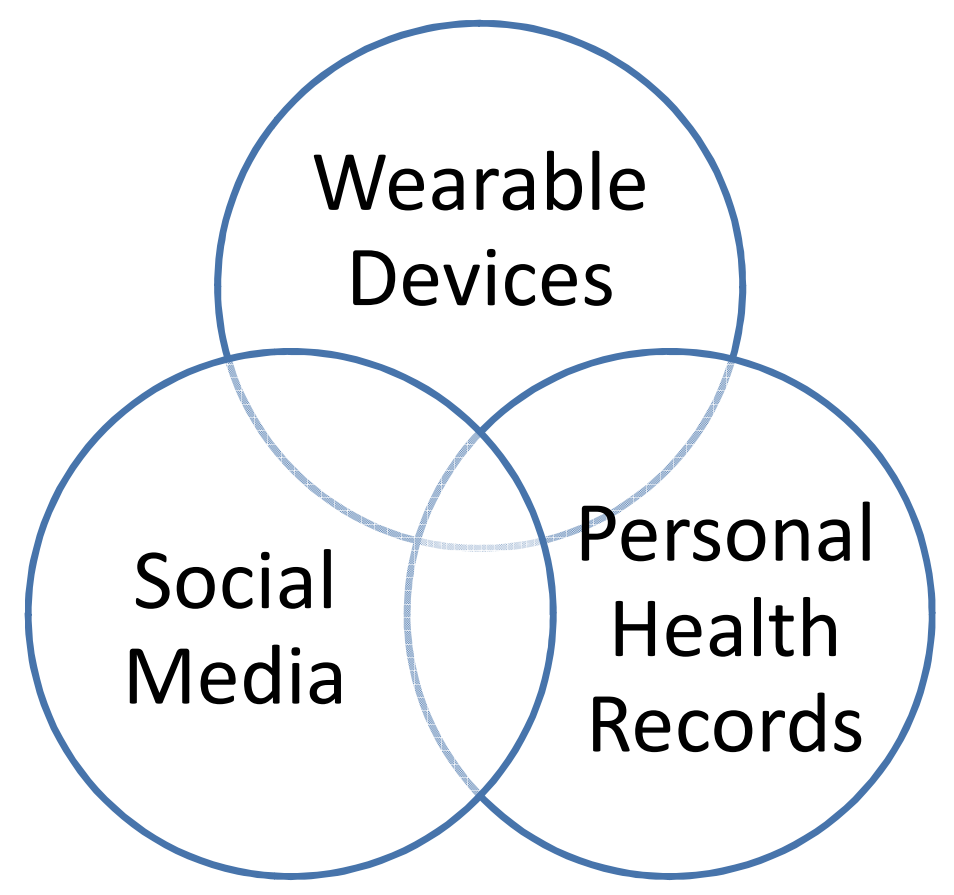

Figure 1: Three intersecting trends enabling self-tracking

\section{Wearable Devices}

As predicted by Intel co-founder Gordon Moore, the density of transistors on microchips has been growing at an exponential rate since the integrated circuit board was invented in the late 1950s resulting in ever increasing computer power in ever smaller devices (2). Thanks to this trend, we now have a wide range of computing devices with embedded high-capability sensors that can record a 
variety of biometric and environmental data including heartbeats, footsteps, GPS locations and even blood sugar levels (3). When plotted against time and space, owners of this hardware can start to see information about their daily activities, fitness regimes, and even about how well they are managing their health conditions.

\section{Social Media Websites}

Concurrent with these developments, there has been a rise in the use of social media websites by patients to monitor their health and fitness (4). Sites such as "Patients Like Me" (5) and "Tu Diabetes" (6) allow community members to enter a variety of data associated with their health state for selftracking over time and for comparison with other community members.

Since the early 2000s, internet interventions have been developed and evaluated across a range of different health domains including asthma (7-11), type 2 diabetes mellitus (12), arthritis, hypertension (13) and mental health $(14,15)$. A Cochrane review of 124 papers concluded that computer-based Interactive Health Communication Applications (IHCA) can improve cognitive and social support outcomes in patients with chronic conditions (16). One of the primary uses of internet interventions in healthcare has been to promote health behaviour change (17). For example, web-based personalised coaching programs with interactive monitoring and tailored feedback have been implemented to promote physical activity (18), assist with smoking cessation (19), and support long-term maintenance of weight loss (20). Significant lifestyle changes have been found to be associated with the use of interactive internet interventions, such as increased exercise time, increased knowledge of nutritional status, slower health decline, and improved body shape perception (21).

Among young people, a recent systematic review concludes there is growing evidence that internetbased self-management interventions can significantly improve selected outcomes in certain childhood 
illnesses such as recurrent pain, obesity, persistent asthma, self-management of brain injury, and encopresis (22). A recent Pew Internet survey, "The Social Life of Health Information", revealed 27\% of the respondents who use the Internet claim they have "tracked their weight, diet, exercise routine or some other health indicators or symptoms online" (4).

\section{Personal Health Records (PHRs)}

Electronic Personal Health Records (PHRs) that allow patients access to their own personal health data have been noted by the Institute of Medicine (IOM) in its recent landmark report to be one of three major consumer IT developments (23). The PHR forms a crucial component in many large-scale national E-Health reform strategies worldwide delivery (Australia: \$467 million; US Obama administration: US\$19 billion; England NHS: $£ 12$ billion). Trials of PHRs have been conducted in in-vitro fertilization (IVF) (24), hypertension (25), diabetes (12), and influenza (26) with emerging evidence on its significant benefits to consumer health outcomes and behaviours (12). In light of the likely future importance of PHRs, the IOM has recommended that they should be monitored from 2012 and subject to FDA regulation if safety and reliability are found to be inadequate (23).

\section{Methods}

In order to examine the implications of these trends, we conducted a review of the literature (both white and grey) of self-tracking and self-experimentation research combined with an environmental scan of popular and current products and services in this area. The result of the literature review were then discussed and interpreted by members of the IMIA Social Media Working Group to formulate the framework presented below.

\section{Results}


The results of the literature review and environmental scan informed the development of a three level framework (see Figure 2) to demonstrate how self-tracking information is gathered and shared.

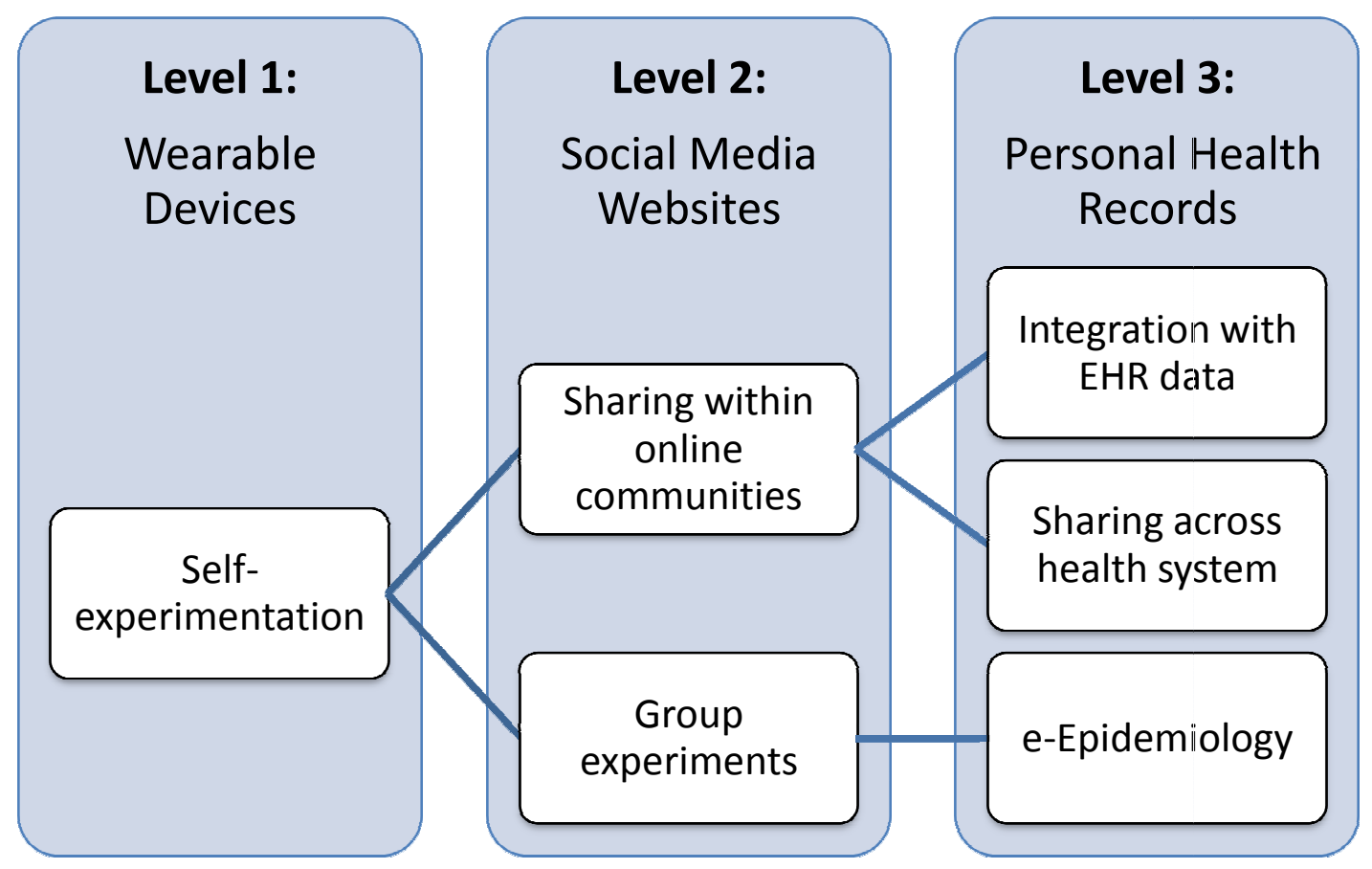

Figure 2: Three level framework for self-tracking systems

Each level represents the trends described in the introduction: Wearable Devices, Social Media Websites and Personal Health Records. Rising through the levels, new issues and research questions have emerged as users move from "Self-Experimentation", where they use devices to provide individual feedback in Level 1, to "Sharing within Online Communities" in Level 2, where users leverage the social web to participate in "Group Experiments" and share their data with a wider community. In Level 3, we see Personal Health Records enabling users to share their data, not just with an online community, but with their healthcare provider through "Integration with EHR data", which is then able to be "Shared across the health system". As we move from level to level, the experimentation moves from the individual, to the group and finally to whole populations where the data becomes part of a wider scientific data set that can enable "e-Epidemiology" for whole populations. 


\section{Level One: Wearable Devices}

Level 1 indicates devices and systems that are primarily designed for personal use. This level includes simple monitors that just provide a one-off reading (a home blood pressure monitor, for example) but also includes devices that store and display historical data, and devices that upload data to a website for viewing online. Some examples of Level 1 devices include:

- BodyMedia (http://bodymedia.com) has produced a disposable patch that is designed to be worn for seven days. It records for each wearer more than 5,000 data points per minute and uses this data to calculate the number of calories burnt, the number of steps taken, and the wearer's activity and sleep patterns.

- The Zeo Sleep Manager (http://myzeo.com/) is a sleep tracking system that incorporates a headband that the user wears while sleeping with a wireless connection to the user's smartphone.

- Fitbit (http://fitbit.com) is a portable sensor that tracks steps taken, stairs climbed and calories burned, and is designed to motivate users to get fit.

- The Wii game console by Nintendo (http://www.nintendo.com/wii/) is an interactive and motion controlled game platform that individuals use for self, social and active entertainment. A Wii Fit and Wii Fit Plus (http://wiifit.com/) platform are also available that include tools and exercise programs for individuals to add to their fitness regimen. The Wii Fit Plus offers a balance board, various physical activities for all ages, multi-player potential, and users can track their physical progress and the number of calories burned each time they interact with the system. 


\section{Level 1 Evidence Base}

Many devices in the Level 1 category have not been subjected to independent evaluation as they are intended, not as scientific instruments or medical devices, but simply as consumer durables. Some of the more advanced Level 1 systems, however, are starting to be used in scientific studies and may even have a place in managing medical conditions. For example, a recent evaluation of the Zeo Sleep monitor concluded that the device has a reasonable level of accuracy and could be used as an alternative to traditional sleep monitoring systems in certain situations (27). As can be seen in our case study, described below, the Nintendo Wii is starting to be used in a wide variety of healthcare settings, and evaluation studies on the efficacy and effectiveness of Wii are emerging $(28,29)$. The makers of FitBit have also participated in a range of studies to validate their device. Dannecker et al (30) evaluated the FitBit device to assess the accuracy of its Energy Expenditure (EE) calculations and found that it is accurate as long as there is correct classification of activities by users. Montgomery-Downs et al (31) found that Fitbit was a suitable tool for monitoring sleep disorders in normative populations but required validation before it could be used diagnostically.

\section{Scientific Experiments at Level 1}

Although the products and services in Level 1 of our framework are often relatively simple and may not integrated into traditional health IT systems, they are none-the-less capable of being used by individuals in a reasonably rigorous fashion. Indeed, patients and physicians have been using personal, unconnected "self-tracking" and "self-experimentation" as a routine part of medical care for many decades (32). Some of the more scientific approaches have been described by Guyatt et al. (33) under the label of " $\mathrm{N}$ of 1 " trials. These trials take an individual patient through a number of treatment options and techniques such as randomisation and blinding to determine whether or not a new treatment works 
over a placebo or a different treatment option. Slick has proposed incorporating " $\mathrm{N}$ of 1 " trials into online tools for helping patients (and physicians) to manage their self-tracking and experimentation to achieve their optimal wellbeing (34).

\section{Level 2: Social Media Self Tracking Communities}

Level two of our framework refers to systems that enable self-trackers to compare their results with other users in online communities. Level 2 systems include websites such as PatientsLikeMe and CureTogether that enable self-trackers to form groups and share their data either privately to a closed group or to the wider public through the community's web pages. The information uploaded by users to these systems is also used for "secondary" purposes to form the basis of group experiments and studies. Many of the devices in the Level 1 category can be combined with Level 2 services such as logging onto a community portal provided by the manufacturer, or through being used by members of an independent networking site. Examples of Level 2 services include:

- PatientsLikeMe ${ }^{\mathrm{TM}}$ (http://patientslikeme.com) is an online community website that allows patients to track a wide variety of health data for a number of conditions. As well as having their own personal profiles, users may compare their results on aggregated charts so they can see how their data measures against other users of the site. The site also offers users a large support community to discuss issues relating to their conditions (35).

- Tu Diabetes (http://tudiabetes.org) is an online community operated by the Diabetes Hands

Foundation which offers support for "people touched by diabetes". The site uses the Ning ${ }^{\mathrm{TM}}$ social networking platform (http://ning.com), and allows users to upload data about their blood results (HbA1c) to be aggregated with other users' data. 
- CureTogether (http://curetogether.com) is a social media website that allows users to share self-reported ratings on a wide variety of disease conditions and management options. The site is focused on quantitative data that is uploaded by its users.

- Run Keeper (http://runkeeper.com) offers users the ability to record maps and other data relating to "runs" they have completed. In addition to data collection, the site offers facilities for the users to network, compare running times, and offer support to each other to keep on top of fitness regimes.

- Moodscope (http://moodscope.com) is a new social networking community that allows users to use online tools to track their mood.

\section{Level 2 Evidence Base}

Evidence of the effectiveness of these communities has increased in recent years. For example, although independent longitudinal evaluations have not yet been conducted, qualitative feedback gathered by PatientsLikeMe administrators and researchers offers a number of suggested benefits (23). An evaluation of the TuDiabetes website conducted in 2010 demonstrated that $81.4 \%$ of users were willing to share $\mathrm{HbA1c}$ data and that the data closely resembled aggregate 2007-2008 estimates from National Health and Nutrition Examination Survey (NHANES) (24). The CureTogether website claims to have data on over 590 conditions and, although would could not find a formal evaluation on its efficacy or health benefits in the academic literature, the site administrators regularly publish infographics generated from the site data on the company blog. Run Keeper has been used in a number of trials as a method of tracking activities levels (31). However, due most-likely to the rapid development of this field, no studies have been found that evaluated the effectiveness of this intervention for sustained health improvement. 


\section{Scientific Experimentation at Level 2}

At Level 2, some of the more complex issues around self-experimentation begin to arise. As data is collected from groups of users, the information begins to resemble more traditional scientific trials, although there are some important differences.

\section{Blinding, Randomisation and Data Collection}

Data collected and aggregated in an ad hoc manner may not be as robust as data collected through the traditional recruitment and data collection methodologies used in standard experiments conducted by researchers. Issues with randomisation and blinding (or the lack thereof) in online self-reporting compared to traditional research methodologies may leave the results open to systematic bias and there may be problems applying standardised statistical tests. Without a robust means for statistical comparison, users of self-tracking communities who view the aggregated data from other users as a benchmark for their own progress may be either overly optimistic or overly pessimistic about their own progress if the aggregated data is systematically biased in a particular direction.

\section{Feedback Loops and the Self-Fulfilling Prophecy}

In addition, there is a potential issue of the aggregate data influencing the reporting of symptoms by individual users and possibly increasing placebo and nocebo effects. As the data generated by individuals is included in the aggregate data pool, this may, under some circumstances, cause the data to continue to trend through the effects of positive and negative feedback mechanisms. The extent to which this might happen could depend on how susceptible the symptoms being reported are to psychological influence. For example, if a group of users start reporting negative mood as a side effect of a new drug, other users watching the graphs of mood tumble over time may place an over-emphasis on their own low mood which they may then report back to the site. This new data point might never have been 
entered if the user hadn't reviewed the negative data already uploaded. This additional report would then drive the graph down further and may then influence more users to report their own mood as low in a negative feedback loop.
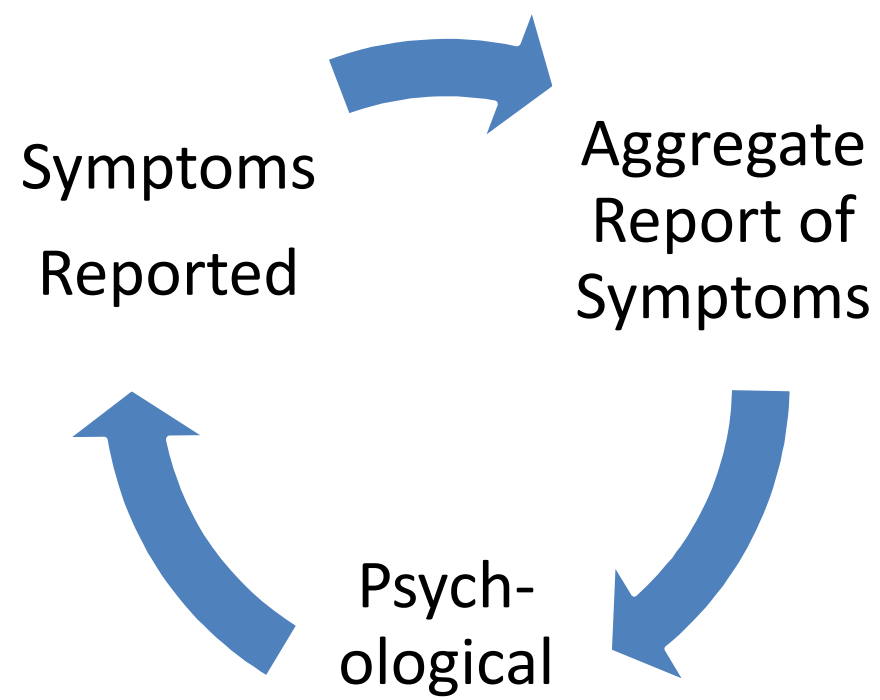

Psych-
ological

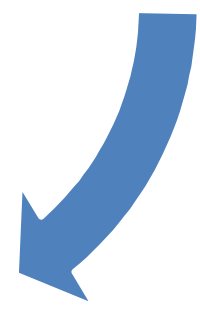
Impact of Report

Figure 3: Self-fulfilling Prophecy Feedback Loop

In addition, for some conditions, the support from the community and the access to data may itself be a significant intervention that could improve the health and/or fitness of the user. In this case, reported aggregate data in these communities may actually be significantly different from individuals who joined more recently and may not be benefiting as much as most other members. Thus, these users may perceive themselves to be not performing as well as others which may have a negative impact on their motivation and outcomes.

We should note that we have not found any evidence of these feedback effects in the online communities we have looked at, but the possibility of this kind of "self-fulfilling prophecy" has been 
found in other social science data-sets (36) and should be investigated further in online health communities.

\section{Self-tracking data and data mining}

Data-mining has enabled website producers to continuously adapt their content according to previous user input such as search queries, comments on blogs, photos and videos uploaded, which groups users join, and the geo-location and device type of the user. In many cases, this information is stored for months or years in order to model users and provide personalised content (for example, a tailored advertisement that matches their interests). Many computer scientists in academia and industry are working on data mining techniques to make sense of the large amount of data that is being generated by internet use, including geo-location from mobile phones.

Large quantities of information can be gathered by analysing web usage data in the health domain (37). For example, uploaded photos may be used to track gender, age and even emotions (38). Natural language processing has been applied to recognize emotions from free text, such as automatic classification of suicide notes in Facebook (39) (40). Links within online social communities can be used to identify leading users, social cues and sub-communities (41).

An example of the potential of such data mining approaches is research-oriented online communities. For example, PatientsLikeMe.com uses natural language processing to analyse forum posts and identify adverse effects of pharmaceuticals (42). The genetics-based online community 23andMe.com (cofunded by Google) is using quiz-like technology to study phenotype information of members who are sharing genetic information. 
In the public health domain there are also many potential applications. It is well known that users of online communities share health risk behaviours such as drug abuse (43). Based on those findings, the Centers for Disease Control (CDC) explored the use of data mining of user profiles to tailor a public health intervention aiming at increasing the effectiveness of online public health interventions (44).

It is hoped that over the next few years the results from self-reported aggregated data will be able to contribute significantly to the research evidence base through new data-mining and statistical methodologies which can control for some of the methodological issues described above whilst taking advantage of the explosion of health-related data being generated on the internet.

\section{Level 3: Integrated Personally Controlled Electronic Health Records}

Level 3 systems in our framework align with the Clayton Christensen concept of the "Patient Controlled EHR", conjectured to be the "disruptive innovation" that has the potential to change the way healthcare is delivered (45). Level 3 indicates systems that utilise health informatics standards to enable aggregation of personally collected data across the health continuum. These systems allow the two way flow of information from individuals to the healthcare system and from healthcare providers to the individuals. Although these systems take the form of a website or online service, they actually represent an ecosystem that consists of hardware devices, adherence to health informatics standards and connections to other health IT systems such as EHRs and Patient Administration Systems (PAS). 


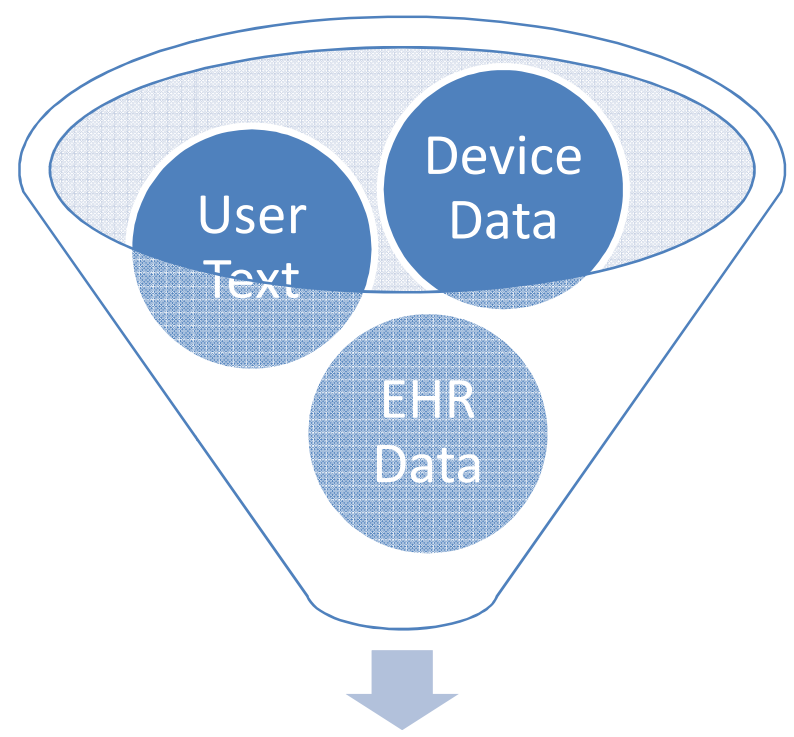

PHR

Figure 4: Multiple Data Sources Contribute to the PHR

There have been several large national initiatives that have aimed to create PHRs for individuals including the NHS's HealthSpace and Australia's PCEHR. Both of these systems have been the subject of considerable debate in the health informatics community $(46,47)$. PHRs from private organisations include Google Health (now closed), Microsoft HealthVault, Patients Know Best and wide variety of products from EHR vendors that act as a "portal" that patients can use to log in to the EHR to view their health information. In general, PHRs to date have focused on one-way flow of information from the health system to the individual, with limited options usually around administrative tasks such as booking appointments. Our environmental scan shows that there does appear to be interest from the wearable device companies for greater integrations with health systems and this might prove to be the driving force between fully connected PHRs we envision in Level 3 of our framework.

\section{Scientific Experimentation at Level 3}


Although the primary purpose of a PHR is to allow the patient, their family and their healthcare providers to access all their health data, it can also be used to contribute to scientific experiments through "Secondary Use".

Secondary Use of health data normally refers to use of EHR data gathered by hospitals and healthcare providers (1), and does not necessarily include data gathered from individuals, whether uploaded manually (as has been the case in many PHRs to date) or collected automatically from self-tracking tools. Tools such as HealthVault (48) enable this automated data collection but we have not yet seen the widespread adoption of tools that combine self-tracking data from wearable devices, data from social media communities with data from hospital information systems. One example of this level of connectedness, however, can be seen in the FitBit device. Data collected from FitBit is now available to multiple third party services including Microsoft HealthVault, which could enable integration with a Personally Controlled EHR. The FitBit user could act at all levels of the framework from simply accessing their own data, to sharing with an online community to using the data in consultations with their doctors.

The applications of bringing together all health data, whether produced by individuals or healthcare systems are potentially significant. At present, many data-sets are limited to periods of hospitalisation or out-patient visits. Combining self-tracking data could greatly increase the contiguousness of the data resulting in far greater accuracy when data-mined. Traditional research data-sets are also heavily weighted towards individuals with on-going health problems. Data from self-tracking healthy populations would add significantly to "control" groups in data-mining exercises and help to increase the specificity of queries run against the data-set. Comparing an individual with a single disease or 
symptom with a completely healthy individual could provide more insight than comparing two individuals with multiple morbidities.

\section{Data Standardisation}

One of the key hurdles to creating this kind of PHR is a lack of adherence to health informatics standards from both the consumer devices and the EHR vendors. In the US, the HITECH ACT is encouraging standards adoption for EHR vendors (49), but to date, there has been relatively little adoption of health informatics standards by self-tracking device manufacturers and social media community providers. This is not surprising as most of these tools are health and fitness orientated and not specifically designed to work with healthcare providers systems.

As it is unlikely that a single provider could encompass hospital systems, social media websites and selftracking wearable devices, the widespread adoption of health informatics standards is essential if we are to achieve the Level 3 PHRs described in our framework.

\section{Privacy and Security Issues}

Another reason for the lack of integration across the levels may be due to the privacy and security provisions that are required when data is collected and stored by traditional healthcare providers. There are serious consequences for data breaches in most developed countries and legal compliance codes that providers must work towards.

In the United States, Title Two of the Health Insurance Portability and Accountability Act (50) has provisions for the security and privacy of patients' health data and the use of electronic data submission across providers and patients. 
More recently, the enactment of the United States Health Information Technology for Economic and Clinical Health Act (HITECH Act) of 2009 reminds health care providers (HCP) to pay close attention to the privacy and security of health data in electronic submissions. In order to be in accordance with Subtitle D of the HITECH Act, a HCP and/or researcher needs to be aware that if "unsecured" protected health information is sent electronically, it would be considered a breach of the HITECH Act. Furthermore, meeting the legal requirements of the HIPAA has been an expense to HCP; if self-tracking data is included under the Act, there could be an added expense for the consumer due to costs in data storage, security, communication, and analyses.

However, most of the self-tracking communities outlined in this article are not provided by traditional healthcare providers and it is usually the patients themselves that are uploading their data. Where they use automated tools, it could be argued they are implicitly consenting to their data being uploaded by the tools to the servers of the company providing the device. Greater clarity of these issues wold both reassure patients as to which systems are and are not secure, and ensure that providers of self-tracking communities handle their data with care.

One way through this difficult legal and ethical terrain might be to keep self-tracking and selfexperimentation data stored in traditional health provider systems that are already monitored for compliance with health privacy laws. Connecting patient communities to healthcare providers system in a Level 3 PHR through standards-compliant secure messaging services (such as HL7) could give providers access to more self-reported data and could enable the providers of self-tracking communities to offer more secure storage and analysis of the health data they collect. 
We have not been able to find any examples of adverse consequences of storing users' health data in communities or repositories associated with self-tracking devices, but we feel this is a topic that will need to be tackled in order to prevent possible negative consequences in the future.

\section{Regulation of Medical Devices}

A recent Pew survey has identified the rapid uptake of mobile technology by healthcare consumers and that this trend is continuing (4). This shift to the mobile device may have the potential to trigger a range of new compliance issues for developers hoping to further integrate consumer devices with patient record systems. For example, some self-tracking applications that are connected to medical devices may fall under the classes of mobile applications that require US Food and Drug Administration (FDA) approval (51). These include: i) mobile applications that are used as an accessory to a regulated medical device (e.g. performing an analysis or process for diagnosis based on patient data); and ii) mobile applications that transform a mobile platform into a regulated medical device (such as connecting the mobile platform to vital signs monitors) (51). Although self-tracking applications embedded with medical devices have the potential to improve consumer health outcomes, the efficacy and safety of these mobile applications needs to be properly established through rigorous testing to ensure compliance with FDA standards in the US and, it is anticipated, counterpart organisations around the world.

\section{Case Study}

Due to the rapid development of this field, evaluation studies on the efficacy and effectiveness of selftracking applications tend to be more limited than in other more stable areas of health informatics. Therefore, in this section we illustrate the potential of self-tracking applications through a case study, 
examining the journey of how a patient recently diagnosed with Parkinson's disease manages and improves his condition with his healthcare professionals and fellow peers through the use of Nintendo Wii games.

Patient N, 65 years-old with a recent diagnosis of Parkinson's disease, read an article about the cognitive, physiological and psychological benefits associated with the Nintendo Wii game console. He is contemplating asking his daughter to purchase a Wii console for him to try and he has consulted with his physician for her approval. Based on recent reports, his physician supports his desire to try the safe and fun virtual game console and his daughter agrees to purchase the Wii for her father's 66th birthday because she thinks it will motivate him to interact without being conscious of his immediate surroundings. $\mathrm{N}$ is hoping the use of Wii tennis and bowling will strengthen his sense of balance, stabilize his gait, increase his energy level and thereafter he will be more independent in his activities of daily living (ADLs). He admits to feeling a "bit blue" at times and he is hoping interacting and competing with his Wii game opponents will help elevate his mood while providing him the opportunity to interact with others.

N's physiotherapist, Amelia, is very interested in learning about the outcomes associated with $\mathrm{N}$ using Wii because Parkinson's disease is the second leading neurodegenerative disorder (52) in the Western world. They agree he will interact with the Wii tennis for one hour, three times a week, for four weeks and then the Wii bowling at the same regimen. Ameila anticipates N's muscles, joints and reflexes will positively benefit from the virtual game activity because of the interactivity the game affords while keeping track of progress.

$\mathrm{N}$ keeps track of his physical balance, joint flexibility, ability to conduct ADLs and mood levels on a scale of one to 10 in a Google spreadsheet before and after each interaction with Wii bowling and tennis. He 20 
emails the results to Amelia in a secure electronic format provided by his physician and she keeps track of his progress over the agreed upon time. At each home visit Amelia and $\mathrm{N}$ track his physical, mental and psychosocial progress in relation to Wii tennis and bowling. It is noted that N's physical balance is better after the bowling activity compared with the tennis activity. However, his self-reported mood quality is remarkably better following a match of tennis with his opponent. Overall, $\mathrm{N}$ is very pleased with his progress and states it gives him the opportunity to compete against some of his friends using Wii tennis. Amelia notes his spirits have increased and are maintained. Moreover, he is more interested in his own self-care and how he socialises with others using the same game console. $\mathrm{N}$ now wants to purchase the Michael Jackson dance program because he wants to challenge himself and ask a female companion to join him dancing.

\section{Conclusion}

The rapid rise of self-tracking devices and social media communities indicates a latent need by certain groups of consumers to better understand their health and wellness. Qualitative aspects of these communities, such as comparing notes with other members, making friends and forming support groups, may be as important as the quantitative feedback mechanisms that often form the core of the services offered.

Although we have described many exciting and innovative services in this paper, it is important to highlight that the majority of patients are not currently engaged in self-tracking or self-experimentation (4). Although this proportion may grow as technology improves, we feel it is likely to remain a minority of the population as a whole. Automated tracking may become an important tool for doctors titrating patients on new medications or management plans, but the success and compliance of the use of these tools will vary with the enthusiasm present in different patients. 
Over time we hope to see the healthcare provider community take advantage of the innovations that are becoming available through patient-led self-tracking solutions. We also hope that by working together with existing healthcare systems and services, the providers of self-tracking communities will be able to offer their customers tools that meet the high standards of safety and accuracy that we have come to expect from healthcare organisations. As more and more data is recorded through self-tracking devices and social media communities, the potential to use this data for health research and to increase the personalisation of health care provision grows more significant. We have attempted to highlight some of the hurdles that need to be overcome, but the goal of fully integrating personally collected data with data currently held in the healthcare system seems achievable.

\section{References}

1. Safran C, Bloomrosen M, Hammond WE, Labkoff S, Markel-Fox S, Tang PC, et al. Toward a national framework for the secondary use of health data: an American Medical Informatics Association white paper. Journal of the American Medical Informatics Association. 2007;14(1):1-9.

2. Moore GE. Cramming more components onto integrated circuits. Proceedings of the IEEE. 1998;86(1):82-5.

3. Swan M. Emerging patient-driven health care models: an examination of health social networks, consumer personalized medicine and quantified self-tracking. International journal of environmental research and public health. 2009 February;6(2):492-525.

4. Fox S. The Social Life of Health Information, 2011: Pew Internet \& American Life Project, 2011.

5. Wicks P, Massagli M, Frost J, Brownstein C, Okun S, Vaughan T, et al. Sharing health data for better outcomes on PatientsLikeMe. Journal of Medical Internet Research. 2010 January;12(2):e19.

6. Weitzman ER, Adida B, Kelemen S, Mandl KD. Sharing data for public health research by members of an international online diabetes social network. PLoS One. 2011 January;6(4):e19256.

7. Chan DS, Callahan CW, Hatch-Pigott VB, Lawless A, Proffitt HL, Manning NE, et al. Internet-based home monitoring and education of children with asthma is comparable to ideal office-based care: results of a 1-year asthma in-home monitoring trial. Pediatrics. 2007 Mar;119(3):569-78.

8. Jan RL, Wang JY, Huang MC, Tseng SM, Su HJ, Liu LF. An internet-based interactive telemonitoring system for improving childhood asthma outcomes in Taiwan. Telemed J E Health. 2007 Jun;13(3):257-68. 
9. Newman S, Steed L, Mulligan K. Self-management interventions for chronic illness. Lancet. 2004 Oct 23-29;364(9444):1523-37.

10. Rasmussen LM, Phanareth K, Nolte H, Backer V. Internet-based monitoring of asthma: a long-term, randomized clinical study of 300 asthmatic subjects. J Allergy Clin Immunol. 2005 Jun;115(6):1137-42.

11. van der Meer V, van Stel HF, Detmar SB, Otten W, Sterk PJ, Sont JK. Internet-based self-management offers an opportunity to achieve better asthma control in adolescents. Chest. 2007 Jul;132(1):112-9.

12. Grant RW, Wald JS, Schnipper JL, Gandhi TK, Poon EG, Orav EJ, et al. Practice-linked online personal health records for type 2 diabetes mellitus: a randomized controlled trial. Arch Intern Med. 2008 Sep 8;168(16):1776-82.

13. Green BB, Cook AJ, Ralston JD, Fishman PA, Catz SL, Carlson J, et al. Effectiveness of home blood pressure monitoring, Web communication, and pharmacist care on hypertension control: a randomized controlled trial. JAMA. 2008 Jun 25;299(24):2857-67.

14. Andrews G, Cuijpers P, Craske MG, McEvoy P, Titov N. Computer therapy for the anxiety and depressive disorders is effective, acceptable and practical health care: a metaanalysis. PLoS One. 2010;5(10):e13196.

15. Titov N, Andrews G, Schwencke G, Robinson E, Peters L, Spence J. Randomized controlled trial of Internet cognitive behavioural treatment for social phobia with and without motivational enhancement strategies. Aust N Z J Psychiatry. 2010 Oct;44(10):938-45.

16. Murray E, Burns J, See TS, Lai R, Nazareth I. Interactive Health Communication Applications for people with chronic disease. Cochrane Database Syst Rev. 2005(4):CD004274.

17. Webb LT, Joseph J, Yardley L, Michie S. Using the Internet to Promote Health Behavior Change: A Systematic Review and Meta-analysis of the Impact of Theoretical Basis, Use of Behavior Change Techniques, and Mode of Delivery on Efficacy. J Med Internet Res. 2010;12(1):e4.

18. van den Berg $\mathrm{MH}$, Schoones JW, Vliet Vlieland TP. Internet-based physical activity interventions: a systematic review of the literature. J Med Internet Res. 2007;9(3):e26.

19. Balmford J, Borland R, Benda P. Patterns of Use of an Automated Interactive Personalized Coaching Program for Smoking Cessation. J Med Internet Res. 2008 December $17 ; 10(5):$ e54.

20. Stevens JV, Funk LK, Brantley JP, Erlinger PT, Myers HV, Champagne MC, et al. Design and Implementation of an Interactive Website to Support Long-Term Maintenance of Weight Loss. J Med Internet Res. 2008 January 25;10(1):e1.

21. Wantland DJ, Portillo CJ, Holzemer WL, Slaughter R, McGhee EM. The effectiveness of Web-based vs. non-Web-based interventions: a meta-analysis of behavioral change outcomes. J Med Internet Res. 2004 Nov 10;6(4):e40.

22. Stinson J, Wilson R, Gill N, Yamada J, Holt J. A Systematic Review of Internet-based Self-Management Interventions for Youth with Health Conditions. Journal of Pediatric Psychology. 2009;34(5):495-510.

23. IOM. Health IT and Patient Safety: Building Safer Systems for Better Care. Washington, DC: Institute of Medicine. The National Academies Press; 2011 [2012/01/17]; Available from: http://www.nap.edu/catalog.php?record id=13269.

24. Lau AYS, Parker A, Early J, Sacks G, Anvari F, Coiera E. Comparative usage of a webbased personally controlled health management system and normal support: a case study in IVF. electronic Journal of Health Informatics (eJHI). 2012;(accepted: 2012/01/24). 
25. Wagner PJ, Dias J, Howard S, Kintziger KW, Hudson MF, Seol YH, et al. Personal health records and hypertension control: a randomized trial. J Am Med Inform Assoc. 2012 Jan 10.

26. Rosenbloom ST, Daniels TL, Talbot TR, McClain T, Hennes R, Stenner S, et al. Triaging patients at risk of influenza using a patient portal. J Am Med Inform Assoc. $2011 \mathrm{Dec} 1$.

27. Shambroom JR, FÁBregas SE, Johnstone J. Validation of an automated wireless system to monitor sleep in healthy adults. Journal of Sleep Research. 2011:no-no.

28. Nitz JC, Kuys S, Isles R, Fu S. Is the Wii Fit ${ }^{T M}$ a new-generation tool for improving balance, health and well-being? A pilot study. Climacteric. 2010;13(5):487-91.

29. Sugarman $\mathrm{H}$, Weisel-Eichler A, Burstin A, Brown R, editors. Use of the Wii Fit system for the treatment of balance problems in the elderly: A feasibility study. Virtual Rehabilitation International Conference, 2009; 2009 June 29 2009-July 22009.

30. Dannecker KL, Petro SA, Melanson EL, Browning RC. Accuracy Of Fitbit Activity Monitor To Predict Energy Expenditure With And Without Classification Of Activities: 725: Board \#2 8:00 AM - 10:00 AM. Medicine \& Science in Sports \& Exercise. 2011;43(5):62 10.1249/01.MSS.0000402857.55729.ab.

31. Montgomery-Downs HE, Insana SP, Bond JA. Movement toward a novel activity monitoring device. Sleep \& breathing = Schlaf \& Atmung. 2011 October:1-5.

32. Kravitz RL, Duan N, Niedzinski EJ, Hay MC, Subramanian SK, Weisner TS. What ever happened to N-of-1 trials? Insiders' perspectives and a look to the future. The Milbank quarterly. 2008 December;86(4):533-55.

33. Guyatt G, Sackett D, Taylor DW, Chong J, Roberts R, Pugsley S. Determining optimal therapy-randomized trials in individual patients. The New England Journal of Medicine. 1986 April;314(14):889-92.

34. Slick IS. Personalized health experiments to optimize well-being and enable scientific discovery. [cited 2012 17/3/2012]; Available from: http://web.media.mit.edu/ eslick/eslick phd proposal.pdf.

35. Frost JH, Massagli MP. Social uses of personal health information within PatientsLikeMe, an online patient community: what can happen when patients have access to one another's data. Journal of Medical Internet Research. 2008 January;10(3):e15.

36. Merton RK. The self-fulfilling prophecy. The Antioch Review. 1948;8(2):193-210.

37. Fernandez-Luque L, Karlsen R, Bonander J. Review of extracting information from the Social Web for health personalization. Journal of Medical Internet Research. 2011 January;13(1):e15.

38. Cottrell GW, Metcalfe J. EMPATH: face, emotion, and gender recognition using holons. 1990:564-71.

39. Huang Y-P, Goh T, Liew CL. Hunting Suicide Notes in Web 2.0 - Preliminary Findings. Multimedia Workshops, International Symposium on. Los Alamitos, CA, USA: IEEE Computer Society; 2007. p. 517-21.

40. Pang B, Lee L. Opinion Mining and Sentiment Analysis. Foundations and Trends in Information Retrieval. 2008;2:1-135.

41. Vinciarelli A, Pantic $M$, Bourlard $H$, Pentland $A$, editors. Social signal processing. Proceeding of the 16th ACM international conference on Multimedia - MM '08; 2008; New York, New York, USA: ACM Press.

42. Frost J, Okun S, Vaughan T, Heywood J, Wicks P. Patient-reported outcomes as a source of evidence in off-label prescribing: analysis of data from PatientsLikeMe. Journal of medical Internet research. 2011;13:e6. 
43. Moreno MA, Parks MR, Zimmerman FJ, Brito TE, Christakis DA. Display of health risk behaviors on MySpace by adolescents: prevalence and associations. Archives of pediatrics \& adolescent medicine. 2009;163:27-34.

44. Mishra N. Online Social Networking: Can it Be Used for Risk Behavior Surveillance. AMIA Spring Congress. Phoenix, Arizona2008.

45. Christensen CM, Grossman JH, Hwang J. The innovator's prescription: a disruptive solution for health care: McGraw-Hill New York; 2009.

46. Greenhalgh T, Wood GW, Bratan T, Stramer K, Hinder S. Patients' attitudes to the summary care record and HealthSpace: qualitative study. BMJ. 2008;336(7656):1290-5.

47. Liaw ST, Hannan T. Can we trust the PCEHR not to leak? The Medical journal of Australia. 2011;195(4):222.

48. Do NV, Barnhill R, Heermann-Do KA, Salzman KL, Gimbel RW. The military health system's personal health record pilot with Microsoft HealthVault and Google Health. Journal of the American Medical Informatics Association. 2011;18(2):118-24.

49. Classen DC, Bates DW. Finding the meaning in meaningful use. New England Journal of Medicine. 2011;365(9):855-8.

50. Health Insurance Portability and Accountability Act of 1996. Pub. L. No. 104-191; 1996.

51. FDA. Draft Guidance for Industry and Food and Drug Administration Staff - Mobile Medical Applications. Food and Drug Administration (FDA). U.S. Department of Health and Human Services; 2011a [2012/01/17]; Available from: http://www.fda.gov/MedicalDevices/DeviceRegulationandGuidance/GuidanceDocuments/ucm26 3280.htm.

52. Paisan-Ruiz C, Jain S, Evans EW, Gilks WP, Simon J, van der Brug M, et al. Cloning of the gene containing mutations that cause PARK8-linked Parkinson's disease. Neuron. 2004 Nov 18;44(4):595-600. 